\title{
Identification and Comparative Determination of Senkyunolide $A$ in Traditional Chinese Medicinal Plants Ligusticum chuanxiong and Angelica sinensis by HPLC Coupled with DAD and ESI-MS
}

\author{
Tao YI, ${ }^{a, b}$ Kelvin Sze-Yin Leung, ${ }^{a}$ Guang-Hua Lu, ${ }^{a}$ Hao Zhang, ${ }^{b}$ and Kelvin ChaN ${ }^{*, a}$ \\ ${ }^{a}$ Research and Development Division, School of Chinese Medicine, Hong Kong Baptist University; Kowloon Tong, \\ Kowloon, Hong Kong Special Administrative Region, P. R. China: and ${ }^{b}$ West China School of Pharmacy, Sichuan \\ University; No. 17, Section 3, Ren-Min-Nan-Lu Road, Chengdu, Sichuan 610041, P. R. China. \\ Received May 20, 2005; accepted August 2, 2005
}

Using the HPLC/DAD/ESI/MS method, the qualitative and quantitative analysis of senkyunolide A (SA) in the rhizomes of Ligusticum chuanxiong (Rhizoma chuanxiong; CX) and roots of Angelica sinensis (DG) was established. As a result, it was found that SA is a characteristic standard compound for the quality evaluation and chemical differentiation between CX and DG. Methanol was chosen in the preparation of standard solutions and extraction of samples based on the stability data. The identity of SA in CX and DG was unambiguously determined based on the quasimolecular ions in ESI-MS. A comprehensive validation of the method, including sensitivity, linearity, reproducibility and recovery, was conducted using the optimized chromatographic conditions. The linear calibration curve was acquired with $R^{2}>0.999$ and limit of detection $(\mathrm{S} / \mathrm{N}=3)$ was estimated to be $12.5 \mu \mathrm{g} / \mathrm{g}$. The reproducibility was evaluated by repeated sample injection and replicated analysis of samples with the relative standard deviation (RSD) value found within $0.68 \%$. The recovery rates of SA varied within the range of $96.91-101.50 \%$ with RSD less than $2.38 \%$. In the present work, the contents of SA were quantified within $3.94-9.14 \mathrm{mg} / \mathrm{g}$ and $0.108-0.588 \mathrm{mg} / \mathrm{g}$ for 12 batches each of CX and DG. The results demonstrated that SA is a useful standard compound for the quality evaluation and chemical differentiation between CX and DG. The analytical procedure is precise and reproducible and thus suitable for the analysis of a large number of samples.

Key words senkyunolide A; Ligusticum chuanxiong; Angelica sinensis; HPLC/DAD/ESI/MS; Umbelliferae; quality evaluation

The dried rhizomes of Ligusticum chuanxiong HoRT (Rhizoma chuanxiong; $\mathrm{CX}$ ), (Umbelliferae) are one of the major clinically used cardiovascular protective botanic medicines in China. ${ }^{1)}$ Having a reputation for facilitating blood circulation and dispersing blood stasis, this herb is commonly prescribed for the treatment of angina pectoris, cardiac arrhythmias, hypertension and stroke. ${ }^{2-4)}$ The roots of Angelica sinensis (Radix Angelica sinensis; DG), the related umbelliferous medicinal plant, is a common traditional Chinese medicine (TCM) that has been used to invigorate blood circulation for the treatment of menstrual disorders, to modulate the immune system, and as an emollient and laxative for chronic constipation of the aged and debilitated. ${ }^{4,5)}$

The constituents in CX and DG are similar, ${ }^{6-9)}$ including alkylphthalides [e.g., Z-ligustilide, senkyunolide A (SA)], phthalide dimers (e.g., levistolide A, tokinolide B), phenolic constituents (e.g., ferulic acid, coniferyl ferulate), etc.

Owing to their availability, ferulic acid and $Z$-ligustilide (Figs. 1a, b) have usually been chosen as chemical markers for the quality evaluation of CX and DG. ${ }^{10-13)}$ However, attempts to distinguish between these closely related species using the above-mentioned chemical markers have met with little success. To solve this problem, a characteristic compound for distinguishing $\mathrm{CX}$ from $\mathrm{DG}$ is desirable.

Senkyunolide A (SA) (Fig. 1c), a characteristic compound found in both CX and DG, inhibits induced proliferation of aortic smooth muscle cells and decreased blood viscosity. It is usually used for the treatment of arteriosclerosis and is one of the ingredients in cosmetic composition. ${ }^{14-16)}$ Previous research suggested that the total amounts of SA in CX are generally higher than those in DG. ${ }^{16)}$ However, the quality evaluation of CX and DG based on SA is still not confirmed.
The present study mainly focuses on a precise, accurate quantitative method for comparison of SA in CX and DG with UV electrospray ionization (ESI) MS techniques.

\section{Experimental}

Materials and Reagents Samples of CX and DG were collected in a number of cultivation bases in mainland China. The sources of the plant materials are listed in Table 1. The identities of these herbs were confirmed by appearance and microscopic and physiochemical analyses according to the Chinese Pharmacopoeia. ${ }^{1,17)}$ Voucher specimens were deposited in the Herbarium Centre, Hong Kong Baptist University.

Reagent-grade solvents including petroleum ether (bp 35-60 ${ }^{\circ} \mathrm{C}$ ), methanol, and ethyl acetate were purchased from Lab-Scan (Bangkok, Thailand) for the extraction of herbs and preparative TLC. HPLC-grade methanol (Lab-scan) was used as the mobile phase for HPLC. Deionized water was generated from a Milli-Q water system (Millipore, Bedford, MA, U.S.A.). Silica gel $60 \mathrm{~F}_{254}$ preparative TLC plates $(1.0 \mathrm{~mm}$ thickness, $20 \times 20 \mathrm{~cm}$, E. Merck, Darmstadt, Germany) were used for preliminary TLC identification. A semipreparative column (Supelcosil, PLC-18, $21.2 \mathrm{~mm} \times$ $250 \mathrm{~mm}, 12 \mu \mathrm{m}$, Supelco) was used in semipreparative HPLC isolation.

HPLC System and Conditions An Agilent 1100 series HPLC-DAD system comprising a vacuum degasser, binary pump, autosampler, thermostated column compartment, and DAD (Hewlett Packard, U.S.A.) was used for quantitative analysis and UV spectra acquisition. The UV detector was set at the maximum absorption wavelength, i.e., $280 \mathrm{~nm}$, of SA.

For chromatographic analyses, an Alltima $\mathrm{C}_{18}$ column $(5 \mu \mathrm{m}, 4.6 \mathrm{~mm} \times$ $150 \mathrm{~mm}$, Alltech Associates, Inc., U.S.A.) with a compatible guard column

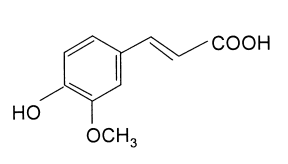

(a)

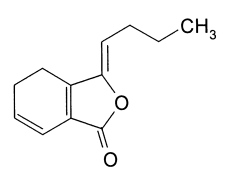

(b)

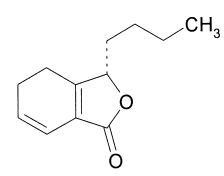

(c)
Fig. 1. Chemical Structures of (a) Ferulic Acid, (b) Z-ligustilide, and (c) Senkyunolide A (SA) 
Table 1. Contents of Senkyunolide A (SA) in 12 Batches of Rhizoma chuanxiong (CX) and 12 Batches of Radix angelica sinensis (DG)

\begin{tabular}{|c|c|c|c|}
\hline Materials & Source & $\begin{array}{l}\text { Year of } \\
\text { harvest }\end{array}$ & $\begin{array}{c}\text { Contents of } \\
\text { senkyunolide } \\
\mathrm{A}(\mathrm{mg} / \mathrm{g})^{a)}\end{array}$ \\
\hline $\mathrm{CX}-1$ & Chongzhou, Sichuan, P.R. China & 2002 & $7.55 \pm 0.15$ \\
\hline $\mathrm{CX}-2$ & Dujiangyanshi, Sichuan, P.R. China & 2003 & $8.43 \pm 0.17$ \\
\hline $\mathrm{CX}-3$ & Chongqing, Sichuan, P.R. China & 2002 & $6.42 \pm 0.12$ \\
\hline $\mathrm{CX}-4$ & Pengzhou, Sichuan, P.R. China & 2001 & $5.90 \pm 0.13$ \\
\hline CX-5 & Chengdu, P.R. China & 2002 & $8.14 \pm 0.14$ \\
\hline CX-6 & Chengdu, P.R. China & 2002 & $5.88 \pm 0.12$ \\
\hline CX-7 & Pengzhou, Sichuan, P.R. China & 2003 & $9.24 \pm 0.16$ \\
\hline CX-8 & Pengzhou, Sichuan, P.R. China & 2001 & $3.92 \pm 0.09$ \\
\hline CX-9 & Chongqing, Sichuan, P.R. China & 2003 & $6.06 \pm 0.12$ \\
\hline CX-10 & Pengzhou, Sichuan, P.R. China & 2002 & $5.67 \pm 0.11$ \\
\hline CX-11 & Dujiangyanshi, Sichuan, P.R. China & 2002 & $5.59 \pm 0.12$ \\
\hline CX-12 & Chengdu, P.R. China & 2003 & $6.82 \pm 0.13$ \\
\hline DG-1 & Minxian, Gansu, P.R. China & 2003 & $0.163 \pm 0.004$ \\
\hline DG-2 & Minxian, Gansu, P.R. China & 2001 & $0.176 \pm 0.005$ \\
\hline DG-3 & Weiyuan, Gansu, P.R. China & 2002 & $0.439 \pm 0.010$ \\
\hline DG-4 & Pingwu, Sicuan, P.R. China & 2002 & $0.226 \pm 0.006$ \\
\hline DG-5 & Pingwu, Sicuan, P.R. China & 2003 & $0.161 \pm 0.004$ \\
\hline DG-6 & Jiuzaigou, Sicuan, P.R. China & 2003 & $0.275 \pm 0.007$ \\
\hline DG-7 & Diqing, Yunnan, P.R. China & 2002 & $0.276 \pm 0.007$ \\
\hline DG-8 & Minxian, Gansu, P.R. China & 2001 & $0.167 \pm 0.004$ \\
\hline DG-9 & Minxian, Gansu, P.R. China & 2002 & $0.305 \pm 0.007$ \\
\hline DG-10 & $\begin{array}{l}\text { Local supplier I, Hong Kong, } \\
\text { P.R. China }\end{array}$ & 2003 & $0.470 \pm 0.010$ \\
\hline DG-11 & $\begin{array}{l}\text { Local supplier II, Hong Kong, } \\
\text { P.R. China }\end{array}$ & 2001 & $0.553 \pm 0.013$ \\
\hline DG-12 & Weiyuan, Gansu, P.R. China & 2002 & $0.177 \pm 0.004$ \\
\hline
\end{tabular}

CX-1 to CX-12 were rhizomes of Ligusticum chuanxiong HorT. DG-1 to DG-7 were the whole roots of Angelica sinensis (OLIv.) Diels. DG- 8 to DG-11 were the heads of $A$. sinensis. DG-12 was a slice of $A$. sinensis. $a)$ The value is mean \pm S.D. $(n=3)$. The S.D. is expressed in three significant figures.

$\left(\mathrm{C}_{18}, 5 \mu \mathrm{m}, 4.6 \mathrm{~mm} \times 7.5 \mathrm{~mm}\right)$ was used. An isocratic elution ( $1 \%$ acetic acid in water: methanol $=40: 60$ ) system was employed. The solvent flow rate was $1 \mathrm{ml} / \mathrm{min}$ and the column temperature was set at $30^{\circ} \mathrm{C}$.

HPLC-MS System and Conditions An Applied Biosystems/PESCIEX API 365 LC-MS system with electrospray ionization source (Applied Biosystems, Foster City, CA, U.S.A.) was used for mass spectrometric determination. The HPLC conditions for HPLC-MS analysis were identical to the one used for HPLC-DAD analysis. The ESI-MS spectrum conditions were optimized in positive-ion mode with the conditions as follows: drying gas air, flow $71 / \mathrm{min}$, gas temperature $300^{\circ} \mathrm{C}$, scan range $50-500 \mathrm{u}$, orifice voltage $26 \mathrm{~V}$, focusing voltage $170 \mathrm{~V}$, and electrospray voltage $5000 \mathrm{~V}$.

Isolation of SA from CX The standard compound SA was isolated as follows: CX powder ( $50 \mathrm{~g}$ ) was extracted under sonication for $30 \mathrm{~min}$ using $300 \mathrm{ml}$ of petroleum ether. The extract was filtered and evaporated to dryness. The residue was then dissolved in methanol and subjected to preparative TLC and semipreparative HPLC for separation and purification. The developing solvents for preparative TLC were petroleum and ether-ethyl acetate $(85: 15, \mathrm{v} / \mathrm{v})$. The mobile phase for preparative HPLC consisted of water (A) and methanol (B) using an optimized gradient elution of $60 \% \mathrm{~B}$ at $0-10 \mathrm{~min}$ and $60-100 \% \mathrm{~B}$ at $10-90 \mathrm{~min}$. The flow rate was $10 \mathrm{ml} / \mathrm{min}$. The detection wavelength was maintained at $280 \mathrm{~nm}$. Purified SA was identified by a comparison of the ${ }^{1} \mathrm{H}$ - and ${ }^{13} \mathrm{C}$-NMR spectra with the published data. ${ }^{18,19)}$ The yield and purity of the isolated SA was found to be $4.57 \mathrm{mg} / \mathrm{g}$ and $97.98 \%$ by HPLC-UV, respectively.

Standard Solution Preparation Accurately weighed $50 \mathrm{mg}$ of SA was introduced into a $50-\mathrm{ml}$ volumetric flask and made up to the volume with methanol as stock standard solution $(1000 \mathrm{mg} / \mathrm{l})$. Aliquots of $0.025,0.1$, $0.25,0.5,1,1.5,2,2.5$, and $3 \mathrm{ml}$ stock standard solutions were transferred into $10-\mathrm{ml}$ volumetric flasks and made up to the volume with methanol as working calibration solutions. The concentrations of SA in calibration solutions were equivalent to $2.5,10,25,50,100,150,200,250$, and $300 \mathrm{mg} / 1$, respectively. An aliquot of $10 \mu \mathrm{l}$ of solution for each calibration was injected for HPLC analysis.

Sample Solution Preparation Sample powder $(0.5 \mathrm{~g})$ was extracted with $8 \mathrm{ml}$ of methanol by means of sonication at room temperature for $0.5 \mathrm{~h}$. The extractions were repeated three times. The total extracts were combined in a $25-\mathrm{ml}$ of volumetric flask and made up to the volume with methanol. The extracts were then filtered through a syringe filter $(0.2 \mu \mathrm{m}$, Alltech, Beerfield, IL, U.S.A.). An aliquot of $10 \mu \mathrm{l}$ of solution was injected for HPLC-DAD and HPLC-ESI-MS analyses. Sample duplicates were prepared as described above for analysis.

\section{Results and Discussion}

Optimization of the Isolation Process for SA Sonication was chosen as the extraction method in the present study. Observation of TLC showed that the amount of SA was more abundant while the polar impurities were minimized using petroleum ether as the extraction solvent. The extract was then subjected to preparative TLC to remove the major extraneous compounds and reduce the load capacity during subsequent semipreparative HPLC purification.

Optimization on the Preparation of SA Stock Solution and Sample Solution $\mathrm{SA}$ is a volatile oil under ambient conditions and therefore its stability remained a technical concern. ${ }^{7)}$ For SA stock solution, its stability was evaluated by comparing the levels of SA in acetonitrile and methanol, respectively, over a period of storage. The levels of SA were found to be $98.7 \%$ and $98.3 \%$, respectively, in acetonitrile and methanol after storing for $5 \mathrm{~d}$, which indicated that SA was relatively stable in both solvent systems.

For the extraction of SA, sonication was chosen as the extraction method for its confirmed efficacy and ease of handling. The choice of extraction solvent for SA in herbs was further compared in methanol and acetonitrile. Persistent turbidity was observed in samples extracted with acetonitrile while the methanolic counterparts remained clear throughout. Petroleum ether was not considered owing to its incompatibility with the reverse-phase HPLC system. Therefore methanol was recommended as the solvent for preparation of standard solution and extraction of SA in herb samples.

Identification of SA in CX and DG by HPLC-MS Apart from comparing with the retention time $\left(t_{\mathrm{R}}\right)$, SA was further identified by HPLC-ESI-MS analysis to provide further information on its identity.

Through comparing MS spectra acquired in negative- and positive-ion modes, the positive-ion mode was found to be more sensitive. For positive-ion mode MS spectra, consistent quasimolecular ions of SA, $[\mathrm{M}+\mathrm{H}]^{+}(m / z=193),[\mathrm{M}+\mathrm{Na}]^{+}$ $(m / z=215)$, and $[\mathrm{M}+\mathrm{K}]^{+}(m / z=231)$ were observed for peak $\mathrm{S}$ in HPLC chromatograms in standard solution, and $\mathrm{CX}$ and DG samples (Figs. 2a, b, c). These findings were in agreement with those reported in the literature. ${ }^{6)}$

Calibration Curves and Limit of Detection SA was quantified in samples using the external standard addition method with a reference marker. Linearity was determined within the concentration range of $2.5-300 \mathrm{mg} / \mathrm{l}$ using nine standard solutions of different concentrations. Linear regression was expressed as $Y=4.885 X+2.37$ with a correlation coefficient of 0.9998 , where $Y$ and $X$ are the value of the area of peak and the concentration of standard solution, respectively. This regression equation was used for quantifying SA in all sample solutions.

The limit of detection (LOD) of SA in samples was determined based on visual evaluation with a signal-to-noise ratio of about $3: 1$. The LOD was estimated to be $0.25 \mathrm{mg} / \mathrm{l}$ in test solution, which was equivalent to $12.5 \mu \mathrm{g} / \mathrm{g}$ in solid samples. 
(a)

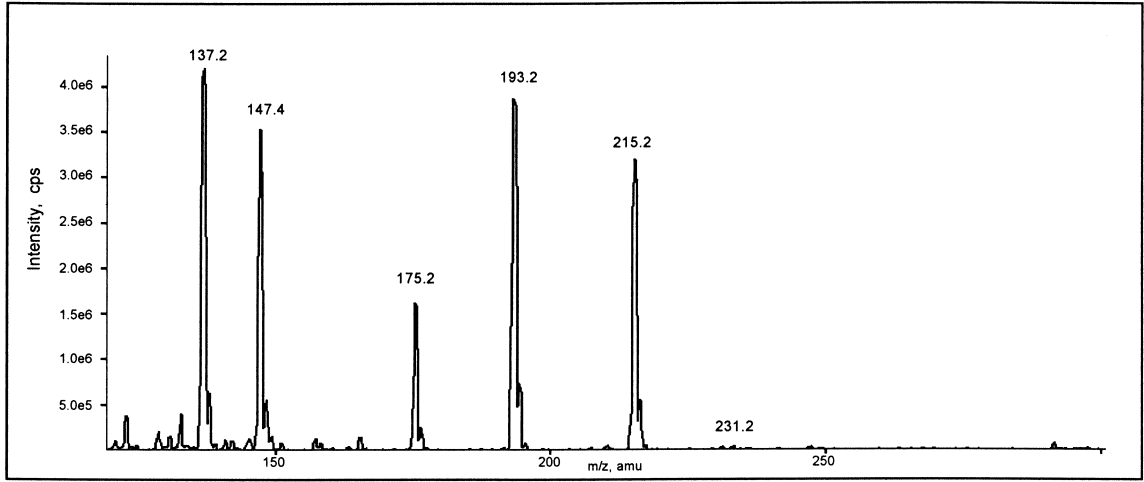

(b)

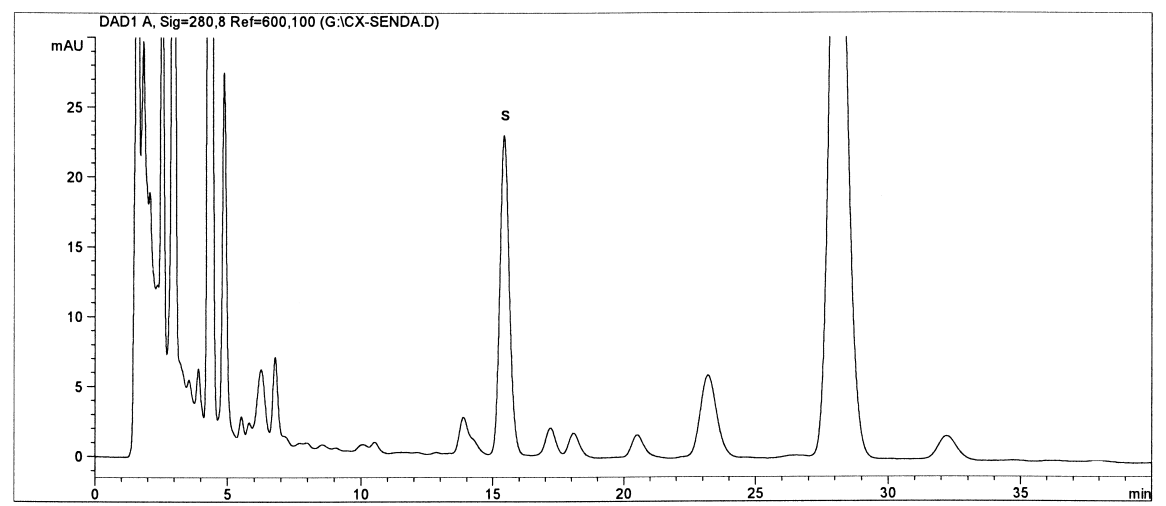

(c)

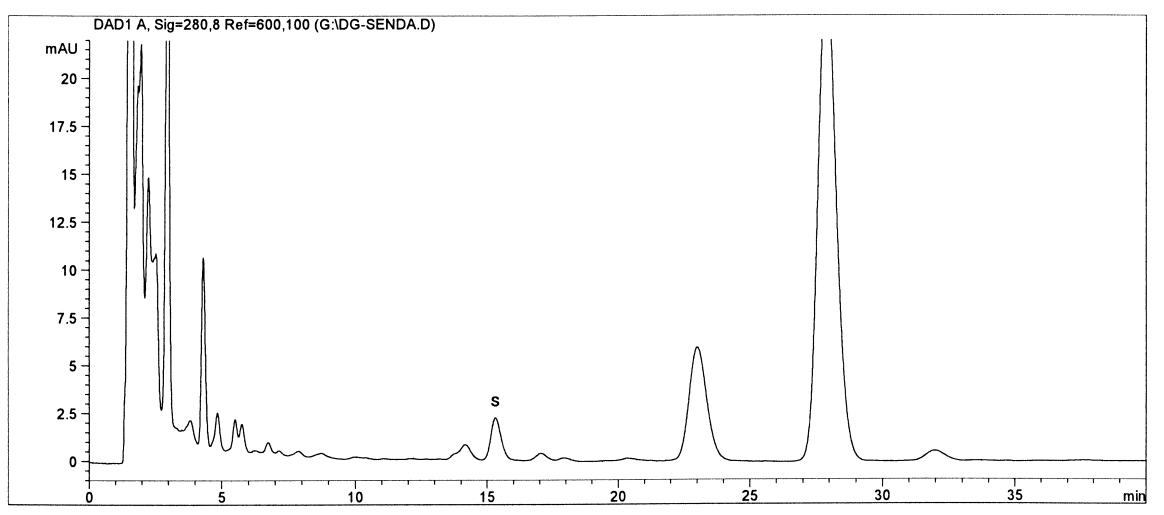

Fig. 2. On-line LC-ESI Mass Spectrum of (a) Senkyunolide A (SA) in Positive Mode, and HPLC Chromatogram of (b) Ligusticum chuanxiong and (c) Angelica sinensis

Moreover, the quantitation limit of SA was determined based on a signal-to-noise ratio of about $10: 1$ for five replicated analyses of spiked matrix blank. The quantitation limit was found to be $0.95 \mathrm{mg} / 1$ in sample solutions, equivalent to $47.5 \mu \mathrm{g} / \mathrm{g}$ in solid samples. These results were considered satisfactory and acceptable for subsequent quantitative analysis.

Method Reproducibility Method reproducibility was evaluated with six repeated injections of standards and six replicated analysis of samples. The precision of replicated injections was determined and the relative standard deviation (RSD) of SA content was found to be $0.25 \%(n=6)$. The RSD of the content of SA in sample replicates was estimated to be $0.68 \%(n=6)$.

Recovery The recovery rate of SA was determined using spiked samples with different concentration levels of $50 \%$, $100 \%$, and $150 \%$ of $\mathrm{SA}$ in the samples. The recovery rates were estimated to be $101.50 \pm 1.54 \%($ mean $\pm \mathrm{RSD}, n=3)$,
$98.12 \pm 1.24 \% \quad($ mean \pm RSD,$\quad n=3$ ), and $96.91 \pm 1.05 \%$ (mean $\pm \mathrm{RSD}, n=3$ ), respectively. The mean recovery was $98.84 \pm 2.38 \%(n=9)$.

Sample Analysis The contents of SA in 12 batches of $\mathrm{CX}$ and 12 batches of DG are listed in Table 1. The contents of SA in CX and DG were within the ranges of 3.94$9.14 \mathrm{mg} / \mathrm{g}$ and $0.108-0.588 \mathrm{mg} / \mathrm{g}$, respectively. On the other hand, the results showed that the content deviation of SA within the same medicinal plant material was also significant. This was probably attributed to the differences in cultivation conditions and processing methods, which gave rise to an inconsistent production of materia medica. However, the content of SA was generally more than 20-fold higher for CX than DG in the present study.

\section{Conclusion}

In this study, the qualitative and quantitative analysis of $\mathrm{SA}$ in rhizomes of $\mathrm{CX}$ and roots of $\mathrm{DG}$ was established using 
HPLC/DAD/ESI/MS. The overall procedure is rapid and reproducible and thus suitable for the analysis of numerous samples.

From the results, the content of SA in CX was generally 20 -fold greater than in DG. Therefore SA is useful as a characteristic standard compound for the quality evaluation as well as chemical differentiation between these two closely related umbelliferic medicinal plants.

Acknowledgment The authors would like to thank Mr. Zhonghui Li (Department of Chemistry, Hong Kong Baptist University) for technical assistance in the NMR analysis.

\section{References}

1) "The Pharmacopoeia of the People's Republic of China," Part I, The Pharmacopoeia Commission of PRC, Chemical Industry Publishing Press, Beijing, 2000, p. 30.

2) Chen K. J., Chen K., Chin. Med. J., 105, 870-873 (1992).

3) Naito T., Kubota K., Shimoda Y., Sato T., Ikeya Y., Okada M., Maruno M., Nat. Med., 49, 288-292 (1995).

4) Hou Y. Z., Zhao G. R., Yang J., Yuan Y. J., Zhu G. G., Hiltunen R., Life Sci., 75, 1775-1786 (2004).

5) Ko W. C., Sheu J. R., Tzeng S. H., Chen C. M., Planta Med., 64, $229-232$ (1998)
6) Zschocke S., Liu J. H., Stuppner H., Bauer R., Phytochem. Anal., 9, 283-290 (1998).

7) Wang W. X., Gu M., Jiang X. G., Gu Z. L., Fan P. S., Chin. Trad. Herb. Drugs, 33, 4-5 (2002).

8) Xiao Y. Q., Li L., You X. L., Masahiko T., Kimiye B., China J. Chin. Mater. Med., 27, 519-521 (2002).

9) Huang W. H., Song C. Q., Acta Pharm. Sin., 38, 680-683 (2003).

10) Ou S. Y., Kwok K. C., J. Trop. Agric. Food Sci., 84, 1261-1269 (2004).

11) Li Y. J., Bi K. S., Biomed. Chromatogr., 17, 543-546 (2003).

12) Zhang H. J., Shen P., Cheng Y. Y., J. Pharm. Biomed. Anal., 34, 705713 (2004).

13) Lu G. H., Chan K., Chan C. L., Leung K., Jiang Z. H., Zhao Z. Z., J. Chromatogr. A, 1046, 101-107 (2004).

14) Kobayashi S., Mimura Y., Naitoh T., Kimura I., Kimura M., Jpn. J. Pharmacol., 63, 353-359 (1993).

15) Ozaki Y., Sekita S., Harada M., Yakugaku Zasshi, 109, 402-406 (1989).

16) Lee K. T., Lee J. N., Jeong J. H., Lee Y. J., Lee S. J., “U. S. Pat. Appl. Publ.," 2004, p. 7.

17) "The Pharmacopoeia of the People's Republic of China," Part I, The Pharmacopoeia Commission of PRC, Chemical Industry Publishing Press, Beijing, 2000, p. 101.

18) Fischer F. C., Gijbels M. J. M., Planta Med., 53, 77-80 (1987).

19) Naito T., Katsuhara T., Niitsu K., Ikeya Y., Mitsuhashi H., Phytochemistry, 31, 639-642 (1992). 